\title{
REGRESSÕES ROBUSTA E LINEAR PARA ESTIMATIVA DE BIOMASSA VIA IMAGEM SENTINEL EM UMA FLORESTA TROPICAL
}

\author{
ROBUST AND LINEAR REGRESSIONS FOR BIOMASS ESTIMATION BY SENTINEL IMAGE IN \\ A TROPICAL FOREST
}

\author{
Aline Bernarda Debastiani ${ }^{1}$, Marks Melo Moura², Franciel Eduardo Rex ${ }^{3}$, \\ Carlos Roberto Sanquetta ${ }^{4}$, Ana Paula Dalla Corte ${ }^{5}$, Naiara Pinto $^{6}$
}

1, 2, 3, 4, 5 Universidade Federal do Paraná, Curitiba, Paraná, Brasil -aline.debastiani@gmail.com, marksmoura@yahoo.com.br, francielrexx@gmail.com, carlossanquetta@gmail.com \& anapaulacorte@gmail.com

${ }^{6}$ National Aeronautics and Space Administration, Pasadena, Califórnia, Estados Unidos da América Naiara.Pinto@jpl.nasa.gov

\section{RESUMO}

A preocupação com as mudanças climáticas globais tem motivado diversos pesquisadores a encontrar métodos eficazes para a quantificação de biomassa florestal e carbono estocado em florestas tropicais, uma vez que, estas atuam de forma mitigatória e compensatória desses efeitos. O sensoriamento remoto tem sido utilizado de forma eficaz e com grande potencial para a estimativas em larga escala, com destaque para dados de Radar de Abertura Sintética (SAR) e imagens multiespectrais. Os estudos desenvolvidos com essa finalidade utilizaram diversas técnicas para associar a biomassa acima do solo (AGB) com os dados obtidos por sensoriamento remoto, entretanto, a aplicação da regressão robusta ainda não está sendo utilizada para tal finalidade. Sendo assim, o objetivo do presente estudo é avaliar o desempenho da regressão robusta comparando com a regressão linear, que é tradicionalmente utilizada, além de avaliar o potencial da utilização dos dados oriundos do satélite Sentinel 1 e 2 para a estimativa de AGB. Neste âmbito, foram utilizadas imagens multiespectrais (Sentinel 2), imagem SAR (Sentinel 1) e como variável resposta a AGB obtida a partir de dados Light Detection and Ranging (LiDAR). A AGB foi estimada por dois métodos de regressão: robusta e linear. Os modelos de regressão robusta e linear apresentaram desempenho semelhante,

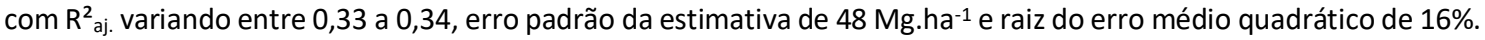
Conclui-se que não houve diferença significativa entre a regressão linear e a regressão robusta para esse conjunto de dados, indicando que a regressão robusta não se sobressaiu com a presença de outliers e que existe potencial na utilização de dados oriundos do satélite Sentinel.

PALAVRAS-CHAVE: Amazônia, Multiespectral, Quantificação, SAR.

\section{ABSTRACT}

Concern about global climate change has motivated several researchers to find effective methods for the quantification of forest biomass and carbon stored in tropical forests, since they act in a mitigating and compensatory way. Remote sensing has been used effectively and with great potential for large scale estimation, with emphasis on Synthetic Aperture Radar (SAR) data and multispectral images. The studies already developed for this purpose have used several techniques to associate above-ground biomass (AGB) with the data obtained by remote sensing, however, the application of robust regression is not yet being used for this purpose. Thus, the objective of the present study is to evaluate the robust regression performance in comparison with the linear regression, that is traditionally used, besides evaluating the potential of the data from Sentinel 1 and 2 satellite. In this context, multispectral images (Sentinel 2), SAR image (Sentinel 1) and as variable response to AGB obtained from data Light Detection And Ranging (LiDAR). AGB was estimated by two regression methods: robust and linear. The robust and linear regression models presented similar performance, with $\mathrm{R}^{2}$ aj. ranging from 0.33 to 0.34 , standard error of the estimate of $48 \mathrm{Mg}$.ha- ${ }^{-1}$ and root mean square error of $16 \%$. It is concluded that there was no significant difference between the linear regression and the robust regression for this data set, indicating that the robust regression did not stand out with the presence of outliers and that there is potential in the use of data from the Sentinel satellite. 


\section{INTRODUÇÃO}

A superfície terrestre abrange cerca de 149,4 milhões de $\mathrm{km}^{2}$, destes, cerca de $30 \%$ são ocupados por florestas (WORLD BANK GROUP, 2018). Os ecossistemas florestais tropicais detém cerca de $40 \%$ do carbono terrestre (PAGE et al., 2009), no entanto, devido ao uso insustentável e desmatamento, o carbono armazenado pode ser liberado na atmosfera como $\mathrm{CO}_{2}$ (dióxido de carbono) contribuindo significativamente para as mudanças no clima. Buscando reduzir a emissão de $\mathrm{CO}_{2}$ e o aquecimento global, alguns países assinaram o Protocolo de Kyoto e o Acordo de Paris, o qual estabelecem metas mitigatórias (MMA, 2018).

Para executar as medidas mitigadoras são necessários sistemas precisos para o monitoramento e quantificação da biomassa florestal em escala nacional e subnacional para os países participantes. Os estoques de carbono florestal são geralmente calculados utilizando a Biomassa Acima do Solo (AGB), assumindo que aproximadamente $50 \%$ da AGB é carbono (GOETZ \& DUBAYAH, 2011). Com isso, o uso do sensoriamento remoto permite a quantificação da AGB e do carbono para uma larga escala espacial, com resultados promissores quando utilizadas imagens multiespectrais ou hiperespectrais (LAURIN et al., 2014; DEO et al., 2017; PHUA et al., 2017), imagens de Radar de Abertura Sintética (SAR) (CASTILLO et al., 2017; SANTI et al., 2017; NINGTHOUJAM et al., 2018) e dados Light Detection and Ranging (LiDAR) (D'OLIVEIRA et al., 2012; NIE et al., 2017; BAZEZEW et al., 2018).

A frequência na utilização e facilidade de cálculo fizeram os mínimos quadrados o método mais popular de análise de regressão linear que alcança bons resultados quando a distribuição de erro subjacente é gaussiana (normal). No entanto, este método não é confiável se o ruído tem componente de média zero ou outliers. Os outliers são valores com comportamento anormal, resultado de desordem das variáveis ou erro de medição (MEER et al., 1991).

Para a detecção e quando confirmada a existência de outliers, é preferível modelos que não considerem essa observação como absoluta, pois atribuiria uma alta influência para essa observação duvidosa. Com esse intuito, na década de 1970 foi desenvolvida a regressão robusta, na qual atribui pesos às observações. Neste sentido, as observações distantes da mediana e que possivelmente sejam outliers recebem menor peso e tem menor contribuição para a modelagem. Esse tipo de regressão é classificado em três categorias principais, sendo: $M$ estimadores, $R$ estimadores e $L$ estimadores. $O$ $M$ estimadores são os métodos de regressão mais populares e tem como princípio minimizar a soma de uma função simétrica dos resíduos (MEER et al., 1991; WILCOX, 2017).

As técnicas de regressão robusta avançaram para o ponto em que podem oferecer ao usuário uma rotina razoável contra os pontos discrepantes e os pontos de alta alavancagem (LI, 1985). Nesse contexto, pelas características das fontes de dados para a modelagem, os pesquisadores utilizam diversos modelos para associar a AGB mensurada em campo com as características das imagens, porém não foi encontrado nenhum estudo que explora o potencial da regressão robusta.

O objetivo do presente estudo é avaliar o potencial da aplicação da regressão robusta comparando com a regressão linear, além de avaliar o potencial da utilização de dados de radar aliados as imagens multiespectrais, para a estimativa da biomassa acima do solo para um fragmento de floresta amazônica.

A hipótese deste estudo é que a regressão robusta apresente desempenho superior ao da regressão linear, com a presença de outliers.

\section{MATERIAL E MÉTODOS}

\section{Aquisição e processamento de dados}

A área de estudo é a Floresta Nacional do Jamari, localizada na região norte do Brasil, no Estado de Rondônia (Figura 1). A fitofisionomia é classificada como Floresta Ombrófila Densa, com algumas porções de Floresta Ombrófila Aberta (IBGE, 2012).

Foram utilizadas as informações extraídas de imagens multiespectrais (satélite Sentinel 2A) e imagens SAR (satélite Sentinel $1 \mathrm{~A}$ ) para relacionar com a AGB obtida a partir de dados LiDAR.

As imagens do satélite Sentinel $1 \mathrm{~A}$ foram coletadas no modo Interferometric Wide Swath com largura de $250 \mathrm{~km}$, nível de processamento na altura do solo (GRD), resolução espacial de $10 \mathrm{~m}$, polarização dupla (FBD), sendo as polaridades VH (transmissão vertical / recebimento horizontal) e VV (transmissão vertical / recebimento vertical), e imageamento na banda C (SENTINEL-1_TEAM, 2013), datadas de 15 de fevereiro de 2016. Mesmo as imagens sendo disponibilizadas com o processamento multi-look (5 x 1), utilizou-se o software SNAP 5.0 (Sentinel's Application Platform) para aplicação do filtro Redefined Lee e redução de Speckle, calibração radiométrica para conversão do valor do pixel em retroespalhamento (Sigma 0, em dB) e correção RangeDoppler Terrain para reprojetar a imagem SAR em UTM. 


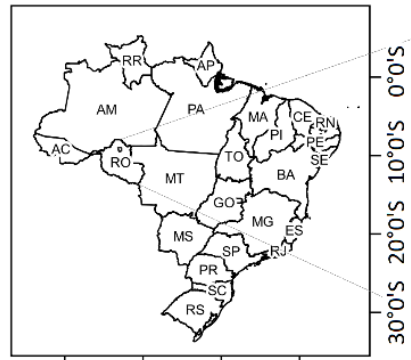

$70^{\circ} 0^{\prime} \mathrm{W} 60^{\circ} \mathrm{O}^{\prime} \mathrm{W} 50^{\circ} \mathrm{O}^{\prime} \mathrm{W} 40^{\circ} \mathrm{O}^{\prime} \mathrm{W}$

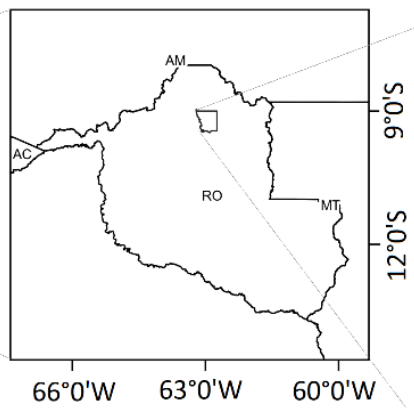

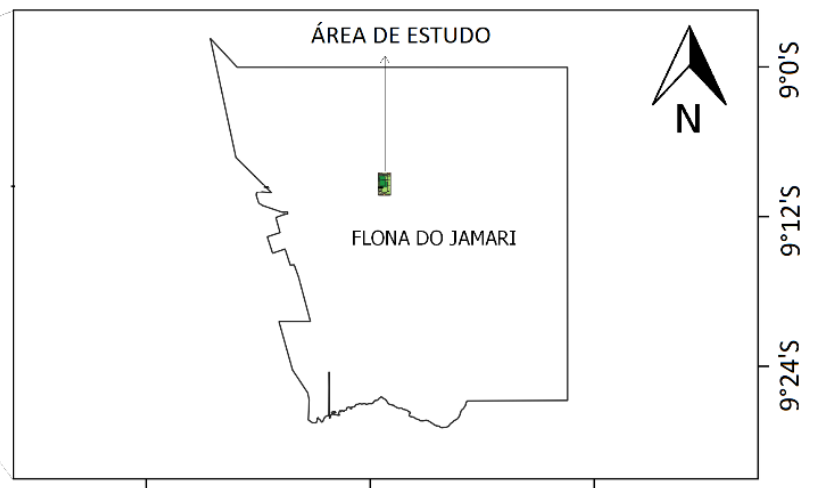

$63^{\circ} 18.0^{\prime} \mathrm{W}$

$63^{\circ} 42^{\prime} \mathrm{W}$

Figura 1. Localização da área de estudo sobre o Brasil, estado de Rondônia e Flona do Jamari.

A fim de se obter informações referentes a estrutura da floresta foram geradas imagens de textura baseadas na Matriz de Co-ocorrência de Nível de Cinza (GLCM) para as bandas VH e VV, sendo as seguintes texturas: contraste, dissimilaridade, homogeneidade, Segundo Momento Angular (ASM), energia, probabilidade máxima (MAX), entropia, média, variância e correlação. As imagens de textura foram geradas com o uso software SNAP.

A imagens multiespectrais do satélite Sentinel 2A foram adquiridas para 25 de agosto de 2015, com nível de processamento $1 \mathrm{C}$ que indicam já terem passado pelo processo de correção geométrica e radiométrica (SENTINEL-2_TEAM, 2015). Para esse satélite são disponibilizadas 13 bandas, sendo quatro bandas com resolução espacial de $10 \mathrm{~m}$, seis bandas com resolução de $20 \mathrm{~m}$ e três bandas com $60 \mathrm{~m}$ de resolução espacial. Realizou-se a correção atmosférica no software QGIS 2.18.16.

Para realçar a vegetação e com o intuito de contribuir com a modelagem, gerou-se quatro índices, sendo eles: Índice de Área Foliar (IAF), Índice de Vegetação da Relação (IVR), Índice de Vegetação por Diferença Normalizada (NDVI) e Índice de Vegetação Ajustado ao Solo (SAVI) considerando 0,25 para a variável de ajuste do solo.

Todos os pixels das imagens oriundas do satélite Sentinel 1 e 2, inicialmente com 10 metros de resolução foram reamostrados para uma resolução espacial de 100 $\mathrm{m}$, para isto, foram utilizados os valores mínimos, máximos, desvio padrão, média e variância de 10 pixels, resultando em um valor para o pixel final de $100 \mathrm{~m}$.

A definição da reamostragem do tamanho do pixel em $100 \mathrm{~m}$ foi baseada nos estudos de Garcia et al. (2018) e Urbazaev et al. (2018), onde os autores testaram várias resoluções e concluíram que a de $100 \mathrm{~m}$ apresentou os melhores resultados.

Os dados LiDAR utilizados para este estudo são oriundos do Projeto Paisagens Sustentáveis do Brasil, o qual recebeu apoio da Empresa Brasileira de Pesquisa Agropecuária (EMBRAPA), do Serviço Florestal do Estados Unidos (USFS), da Agência dos Estados Unidos para o Desenvolvimento Internacional (USAID) e pelo Departamento de Estado dos Estados Unidos. Os dados foram adquiridos para 21 de setembro de 2015 e as especificações do levantamento constam na Tabela 1.

Tabela 1. Especificações do aerolevantamento com LiDAR.

\begin{tabular}{cc}
\hline Características & Especificações \\
\hline Datum & Sirgas 2000 \\
\hline Sistema de coordenadas UTM & $20 \mathrm{~S}$ \\
Área total & $500 \mathrm{ha}$ \\
Média de densidade de & $15,43 \mathrm{ppm}^{2}$ \\
pulsos $/ \mathrm{m}^{2}$ & $850 \mathrm{~m}$ \\
Altitude média do voo & $11,1^{\circ}$ \\
Campo de visão & $59,8 \mathrm{~Hz}$ \\
Frequência de varredura & $65 \%$ \\
\hline Porcentagem de sobreposição &
\end{tabular}

A nuvem de pontos oriundas do levantamento LiDAR foram processadas com o uso do software FUSION 3.60 (MCGAUGHEY, 2016), pela qual uma filtragem de pontos fora gerada para classificar os pontos referentes ao solo e gerar o Modelo Digital de Terreno (MDT), fez-se o mesmo para obter o Modelo Digital de Superfície (MDS) que consiste nos pontos localizados acima da cobertura florestal. A partir da diferença entre os dois modelos obteve-se a altura da vegetação e posterior extração das demais métricas as quais foram aplicadas na equação de D’Oliveira et al. (2012) (Eq. 1) para a obtenção da biomassa seca acima do solo. 
$A G B=(3,119+0,564 P 25+0,062 \text { Var })^{2}+1,74$

Em que: $A G B=$ representa a biomassa seca acima do solo ( $\mathrm{Mg}$.ha-1 ${ }^{-1}$, árvores DAP $\geq 10 \mathrm{~cm}$ ); P25 = equivale aos retornos do primeiro quartil ou $25 \%$ percentil; e Var = variação da elevação.

A equação alométrica de D'Oliveira et al. (2012) considera erro de 40,2 Mg.ha-1, erro esse que deve ser somado ao erro da modelagem para o computo final do erro. A AGB foi obtida também para um tamanho de pixel de $100 \mathrm{~m}$, e consiste no somatório total de AGB.

\section{Modelagem da biomassa seca acima do solo}

As bandas e subprodutos SAR e multiespectrais passaram por um processo de seleção dos pares de variáveis a comporem os modelos de regressão, sendo utilizado o algoritmo CFS (CfsSubsetEval) implementado no software WEKA (Waikato Environment for Knowledge Analysis) para tal finalidade.

O objetivo principal do algoritmo CFS é selecionar as melhores variáveis de acordo com os valores de correlação entre a variável dependente, e excluindo as autocorrelações. Para tal, os subconjuntos de dados foram selecionados usando um algoritmo de busca heurística juntamente com uma função de correlação de Pearson (HALL, 1999).

Após selecionados os pares de variáveis independentes, estes foram relacionados com a $A G B$ obtida do levantamento LiDAR. A relação foi construída utilizando a regressão linear pelo método dos mínimos quadrados (OLS) e dois tipos de regressão robusta, a de Huber e Bisquare (LI, 1985). A regressão foi realizada utilizando o software RStudio e o package MASS (R CORE TEAM, 2017).

Utilizou-se a AGB obtida dos dados LiDAR como variável dependente, motivados pela inexistência de dados de campo e pela confiabilidade das estimativas que estes produtos fornecem.

No total, foram utilizados 286 pixels para ajuste dos modelos. O conjunto de dados não foi subdividido para destinar parte dos dados para teste, com o intuito de observar o comportamento da regressão robusta com a existência de pontos discrepantes de AGB, ou outliers, e compara-lo a regressão linear.

A avaliação do desempenho dos modelos de regressão foi realizada a partir do coeficiente de determinação ajustado $\left(R^{2}{ }_{\text {aj. }}\right)$, erro padrão da estimativa $\left(S_{y x}\right)$ e raiz do erro médio quadrático (RMSE). A fim de constar se existe diferença significativa entre os modelos de regressão ajustados, realizou-se o teste de médias de Tukey no software ASSISTAT (SILVA \& AZEVEDO, 2016).

\section{RESULTADOS E DISCUSSÃO}

O algoritmo CFS selecionou 10 variáveis dentre as que obtiveram maiores correlações com a variável dependente para compor os modelos de regressão (Eq.2). Os coeficientes de ajuste dos modelos de regressão linear e robusta, e o valor de correlação das variáveis com a AGB constam na Tabela 2.

$A G B_{\text {est }}=\beta_{0}+\beta_{1} * \operatorname{Var}(\operatorname{VH} M A X)+\beta_{2} * \operatorname{Var}(V V$ ret. $)+\beta_{3} *$ $\operatorname{Var}\left(V V\right.$ correlação $+\beta_{4} * \operatorname{Var}(S A V I)+\beta_{5} *$

$\operatorname{Var}(\operatorname{banda} 13)+\beta_{6} * \operatorname{Var}($ banda 2$)+\beta_{7} * \operatorname{Var}($ banda 3$)+$ $\beta_{8} * \operatorname{Var}($ banda 4$)$

A maioria das variáveis selecionadas se refere aos produtos oriundos do satélite Sentinel 2, sendo que a variável mais correlacionada com a AGB de acordo com a correlação linear de Pearson é o SAVI $(0,442)$ e a de menor correlação é a textura que indica a probabilidade máxima, representada na forma de variância dentro do conjunto de pixels de $100 \mathrm{~m}$.

Tabela 2. Correlação linear de Pearson ( $r$ ) com AGB e coeficientes de ajuste da regressão linear e robusta.

\begin{tabular}{|c|c|c|c|c|}
\hline \multirow{2}{*}{ Coeficientes } & \multirow[b]{2}{*}{$\mathbf{r}$} & \multirow{2}{*}{$\begin{array}{c}\text { Regressão } \\
\text { linear (OLS) }\end{array}$} & \multicolumn{2}{|c|}{ Regressão robusta } \\
\hline & & & Huber & Bisquare \\
\hline Intercepto & - & $6,10 \times 10^{-5}$ & $6,07 \times 10^{-8}$ & $6,16 \times 10^{-8}$ \\
\hline Var (VH MAX) & $-0,098$ & $-6,55 \times 10^{-6}$ & $-6,40 \times 10^{-9}$ & $-6,18 \times 10^{-9}$ \\
\hline Var (VV ret.) & 0,154 & $1,41 \times 10^{-6}$ & $1,63 \times 10^{-9}$ & $1,70 \times 10^{-9}$ \\
\hline $\begin{array}{c}\text { Var (VV } \\
\text { correlação) }\end{array}$ & $-0,116$ & $-2,69 \times 10^{-10}$ & $-2,72 \times 10^{-13}$ & $-2,71 \times 10^{-13}$ \\
\hline Var (SAVI) & 0,442 & $1,95 \times 10^{-8}$ & $1,57 \times 10^{-11}$ & $1,38 \times 10^{-11}$ \\
\hline Var (banda 13) & 0,283 & $-6,95 \times 10^{-8}$ & $-2,51 \times 10^{-11}$ & $-2,31 \times 10^{-11}$ \\
\hline Var (banda 2) & 0,272 & $5,92 \times 10^{-9}$ & $3,27 \times 10^{-12}$ & $4,14 \times 10^{-11}$ \\
\hline Var (banda 3) & 0,357 & $5,08 \times 10^{-9}$ & $5,67 \times 10^{-12}$ & $5,96 \times 10^{-12}$ \\
\hline Var (banda 4) & 0,301 & $3,42 \times 10^{-9}$ & $3,37 \times 10^{-12}$ & $3,60 \times 10^{-12}$ \\
\hline Var (banda 5) & 0,331 & $-1,01 \times 10^{-9}$ & $-9,01 \times 10^{-11}$ & $-7,75 \times 10^{-11}$ \\
\hline Min (banda 12) & $-0,414$ & $-3,26 \times 10^{-6}$ & $-3,21 \times 10^{-9}$ & $-3,24 \times 10^{-9}$ \\
\hline
\end{tabular}

Var: é a representação da Variância da variável abrangendo o pixel de 100 m ou 10 pixels de 10 m oriundos do Satélite Sentinel 1 e 2; Mín: representa o menor valor da variável dentro do pixel de 100 m; MAX: medida de textura que indica a probabilidade máxima; SAVI: índice de vegetação ajustado ao solo; VH ou VV: bandas SAR; banda 2, 3, 4, 5, 12 ou 13: bandas do satélite Sentinel 2. ret: retroespalhamento. 
O modelo de regressão linear apresentou um melhor desempenho $\left(R^{2}\right.$ aj. $=0,342, S_{y x}=48,355 \mathrm{Mg} \cdot h^{-1}$ e $R M S E=$ $16,005 \%)$ quando comparado aos modelos de regressão robusta. O erro padrão da estimativa foi de $48 \mathrm{Mg}$.ha-1 ${ }^{-1}$ para os modelos, sendo que a média da AGB é de $296 \mathrm{Mg}$ ha $^{-1}$, representando cerca de $16 \%$ de erro (Tabela 3 ).

Tabela 3. Estatística de ajuste e desempenho dos modelos de regressão, avaliados pelo coeficiente de determinação ajustado $\left(R^{2}{ }_{\text {aj. }}\right)$, erro padrão da estimativa $\left(S_{\mathrm{yx}}\right)$ e raiz do erro médio quadrático (RMSE).

\begin{tabular}{cccc}
\hline Regressão & $\mathbf{R}_{\text {aj. }}$ & $\mathbf{S}_{\mathbf{y x}}$ (Mg.ha- $^{-1}$ ) & RMSE (\%) \\
\hline Linear & 0,342 & 48,355 & 16,005 \\
Huber & 0,338 & 48,516 & 16,058 \\
Bisquare & 0,334 & 48,636 & 16,098 \\
\hline
\end{tabular}

Com base na Tabela 3 e Figura 2 é possível constatar que os valores estimados pela regressão linear e robusta apresentaram comportamento semelhante. Acredita-se que os pesos atribuídos pela regressão robusta aos outliers não foi significativamente diferente dos demais pontos da análise, não permitindo um melhor desempenho na modelagem.
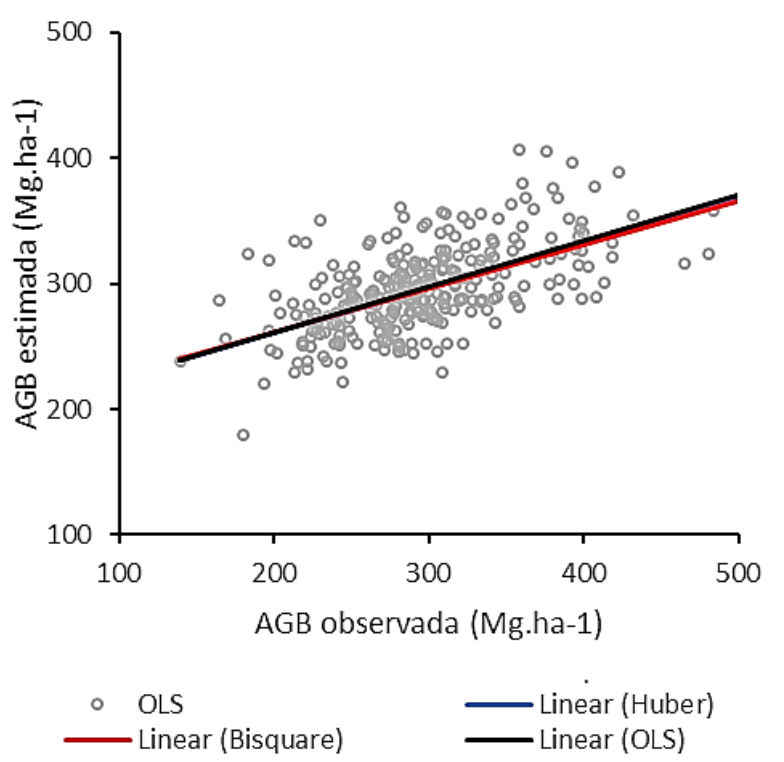

Figura 2. Dispersão dos pontos e linha de tendência entre a AGB observada pelos dados LiDAR em relação a estimada pelas regressões robusta e linear.

Percebe-se que a regressão robusta de Huber foi a que mais atribuiu peso igual a 1 para os valores de $A G B$ mensurados (Figura 3). Para peso superior a 0,8, a regressão de Huber apontou mais de $87 \%$ e para Bisquare mais de $83 \%$ dos dados. Admite-se que estas observações com peso superior a 0,8 são confiáveis, não correndo o risco de serem outliers. Já os menores pesos atribuídos as observações colocam em dúvida o modelo gerado considerando suas observações com mesma capacidade descritiva das demais. A regressão de Bisquare foi a que atribuiu menores pesos, com $1,74 \%$ das observações com peso $<0,2$, e $2 \%$ das observações com peso entre 0,2 e 0,4. Deste modo, percebe-se que a regressão robusta por Bisquare é a mais restritiva e possivelmente a mais confiável, apesar do seu desempenho ter sido levemente inferior a regressão de Huber.

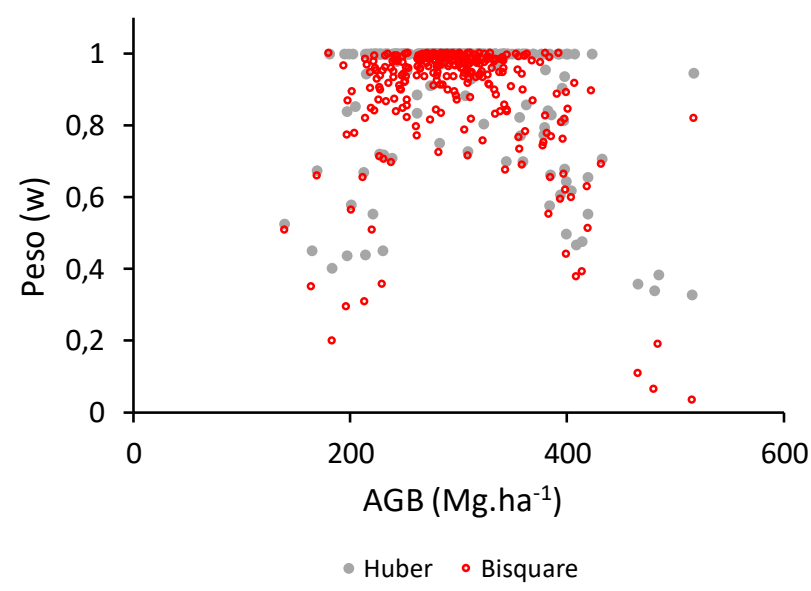

Figura 3. Dispersão dos pesos que os modelos de regressão de Bisquare e Huber atribuíram aos valores de AGB.

$\mathrm{Na}$ Figura 4 é possível observar o comportamento espacial da AGB mensurada a partir dos dados LiDAR e as estimadas pelos modelos de regressão. Assim como observado na estatística de desempenho, o comportamento foi semelhante também para a espacialização dos valores.

O desempenho de ajuste dos modelos de regressão foi inferior aos encontrados por outros autores para a estimativa de AGB a partir de dados SAR e multiespectrais, tais como: Berninger et al. (2018), Castillo et al. (2017) e Nguyen et al. (2016).

Berninger et al. (2018) utilizaram um modelo de regressão linear multivariado e imagens SAR de banda $L$ e C para estimar a AGB de uma floresta tropical da Indonésia para os anos de 2007, 2009 e 2016. Os autores encontram $R^{2}$ variando de 0,63 e 0,77. Esse desempenho superior ao encontrado no presente estudo se deve em partes a utilização da banda $\mathrm{L}$, que proporciona uma maior penetração e caracterização da floresta, comparado quando utilizado apenas a banda $\mathrm{C}$. 


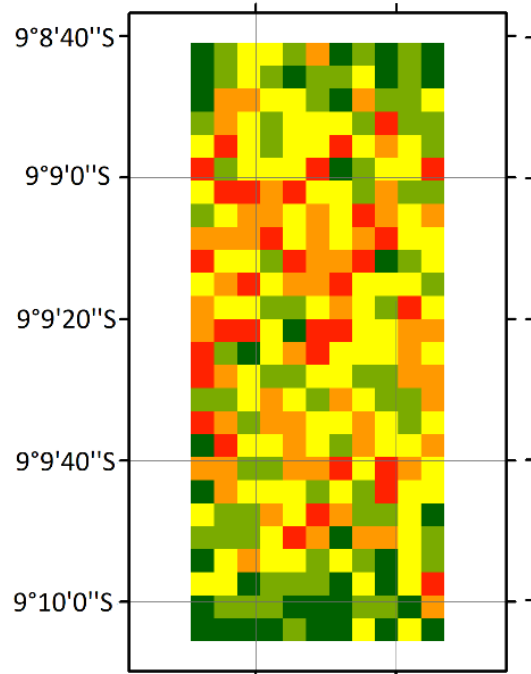

$62^{\circ} 59^{\prime} 0^{\prime \prime} \mathrm{W} \quad 62^{\circ} 58^{\prime} 40^{\prime \prime} \mathrm{W}$

AGB obs

(mg.ha-1) $\square$ 276-318

139-232 317-376

233-275 $377-516$

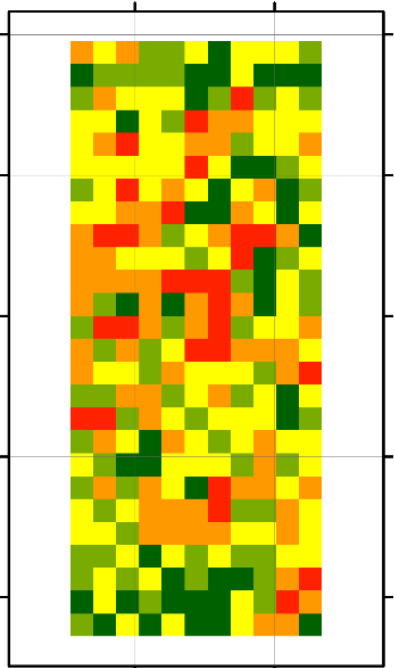

$62^{\circ} 59^{\prime} 0^{\prime \prime} \mathrm{W} \quad 62^{\circ} 58^{\prime} 40^{\prime \prime} \mathrm{W}$

AGB est. OLS

(mg.ha-1) $\square 285-310$

180-259 311-347

260-284 348-448

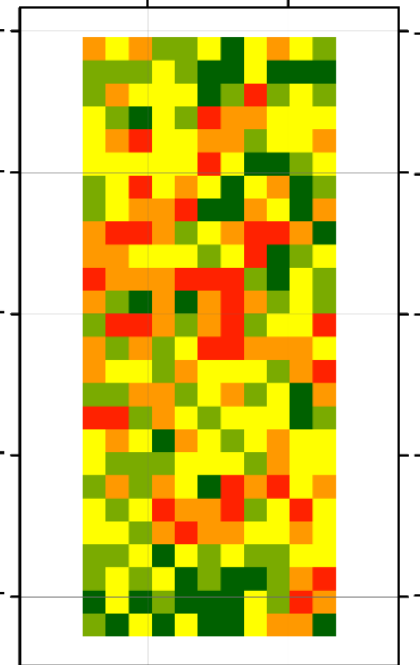

$62^{\circ} 59^{\prime} 0^{\prime \prime} W \quad 62^{\circ} 58^{\prime} 40^{\prime \prime} W$

AGB est. Huber

(mg.ha-1) $\square$ 282-306

180-257 307-339

258-281 340-458

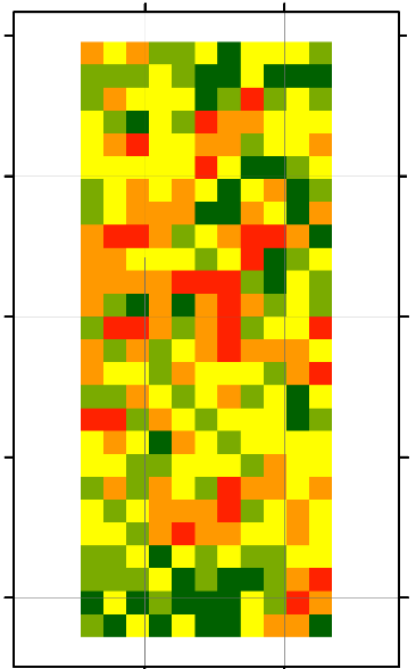

$62^{\circ} 59^{\prime} 0^{\prime \prime} W \quad 62^{\circ} 58^{\prime} 40^{\prime \prime} W$

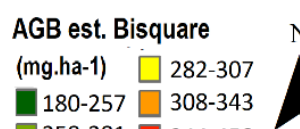

258-281 $344-458$

Figura 4. Espacialização da biomassa florestal aérea observada e estimada por: regressão linear (OLS), regressão robusta por Huber e Bisquare.

Castillo et al. (2017) utilizaram imagens do satélite Sentinel e vários algoritmos de aprendizado de máquina e regressão linear para estimar a AGB de áreas ocupadas por mangue nas Filipinas. Encontraram $\mathrm{R}^{2}$ próximo de 0,67 e

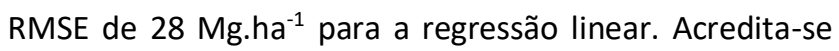
que esse melhor resultado esteja relacionado ao tipo de vegetação estudada, ou seja, a saturação da banda C é determinada primariamente pela densidade da vegetação que no estudo foram os mangues, caracterizado por ser uma fitofisionomia que difere no seu grau de densidade com a floresta Amazônica, portanto possibilita melhor desempenho dos estimadores.

Nguyen et al. (2016) mapearam a biomassa de floresta decídua e tropical no Vietnã. Utilizaram de dados oriundos do satélite Landsat 8 e Alos-2 (banda L), bem como subprodutos oriundos desses satélites (textura, NDVI), além de dados de inventario. Os autores testaram oito modelos com diferentes combinações de variáveis independentes para a regressão linear múltipla, e encontraram $R^{2}$ variando de 0,36 (utilização apenas das texturas SAR) a 0,78 (combinação de imagens do Landsat 8 e Alos-2). O menor RMSE observado pelos autores foi de

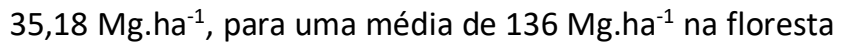

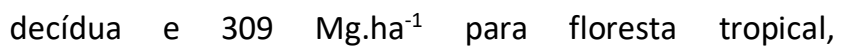
representando $25 \%$ e $11 \%$ de erro, respectivamente. Ao comparar a amplitude do erro relativo encontrado por Nguyen et al. (2016), os resultados do presente estudo estão dentro da amplitude de valores (16\%).

Apesar de não ter ocorrido diferença significativa entre a regressão robusta e linear, recomenda-se aplicar a metodologia deste estudo em outros conjuntos de dados, para validar o comportamento da regressão robusta.

\section{CONCLUSÕES}

Ao considerar as estatísticas de RMSE e $\mathrm{S}_{\mathrm{yx}}$ admite-se que foi possível estimar com precisão a biomassa acima do solo utilizando de imagem multiespectral e SAR oriundos dos satélites Sentinel.

Os dados provenientes do satélite Sentinel demonstraram alta aptidão para a estimativa da AGB para a floresta amazônica devido suas vantagens para mapeamento em larga escala e com baixo custo, já que as imagens são disponibilizadas de forma gratuita.

Entre as estatísticas comparativas da regressão robusta de Huber e Bisquare não houve diferenciação, entretanto na atribuição dos pesos houve uma maior restrição utilizando o modelo de Bisquare.

Os desempenhos dos modelos de regressão robusta e linear apresentaram comportamentos semelhantes, não diferindo estatisticamente. Portanto, a regressão robusta não apresentou melhor desempenho com a presença de outliers, rejeitando a hipótese testada neste estudo.

\section{AGRADECIMENTOS}

O presente trabalho foi realizado com apoio da Coordenação de Aperfeiçoamento de Pessoal de Nível 
Superior - Brasil (CAPES) - Código de Financiamento 001.

Ao Projeto Paisagens Sustentáveis Brasil, da Empresa Brasileira de Pesquisa Agropecuária (EMBRAPA), pela permissão da utilização dos dados LiDAR para elaboração deste estudo. Dados adquiridos com apoio financeiro da Agência para o Desenvolvimento Internacional Norte Americana (USAID) e do Departamento de Estado Norte Americano (USDS) e com apoio técnico do Serviço Florestal Norte Americano (USFS).

\section{REFERÊNCIAS}

BAZEZEW, M.N. et al. Integrating airborne LiDAR and terrestrial laser scanner forest parameters for accurate above-ground biomass/carbon estimation in Ayer Hitam tropical forest, Malaysia. International Journal of Applied Earth Observation and Geoinformation, v.73, p.638-652, 2018.

BERNINGER, A. et al. SAR-Based estimation of above-ground biomass and its changes in tropical forests of Kalimantan using Land C-Band. Remote Sensing, v.10, n.6, p.831, 2018.

CASTILLO, J.A.A. et al. Estimation and mapping of above-ground biomass of mangrove forests and their replacement land uses in the Philippines using Sentinel imagery. ISPRS Journal Photogrammetry Remote Sensing, v.134, p.70-85, 2017.

DEO, R. et al. Using landsat time-series and LiDAR to inform aboveground forest biomass baselines in Northern Minnesota, USA. Canadian Journal of Remote Sensing, v.43, p.28-47, 2017.

D'OLIVEIRA, M.V.N. et al. Estimating forest biomass and identifying low-intensity logging areas using airborne scanning LiDAR in Antimary State Forest, Acre State, Western Brazilian Amazon. Remote Sensing of Environment, v.124, p.479-491, 2012.

GARCIA, M. et al. Modelling forest canopy height by integrating airborne LiDAR samples with satellite Radar and multispectral imagery. International Journal of Applied Earth Observation and Geoinformation, v.66, p.159-173, 2018.

GOETZ, S.; DUBAYAH, R. Advances in remote sensing technology and implications for measuring and monitoring forest carbon stocks and change. Carbon Management, v.2, p.231-244, 2011

HALL, M.A. Correlation-based feature selection for machine learning. New Zealand: Department of Computer Science University of Waikato, 1999.

IBGE. Manual técnico da vegetação brasileira. 2.ed. 2012.

LAURIN G.V. et al. Above ground biomass estimation in an African tropical forest with lidar and hyperspectral data. ISPRS Journal of Photogrammetry and Remote Sensing, v.89, p.49-58, 2014.

LI, G. Robust regression. In: Exploring data tables, trends, and shapes. Wiley, 1985.

MCGAUGHEY, R.J. FUSION / LDV: software para análise e visualização de dados LIDAR. Seattle: USFS, 2016.
MEER, P. et al. Robust regression methods for computer vision: a review. International Journal of Computer Vision, v.6, n.1, p.5970, 1991.

MMA - MINISTÉRIO DO MEIO AMBIENTE. Protocolo de Quioto. 2018. Disponível em: http://www.mma.gov.br/clima/convencaodas-nacoes-unidas/protocolo-de-quioto

NGUYEN, L.V. et al. Mapping tropical forest biomass by combining ALOS-2, Landsat 8, and field plots data. Land, v.5, n.31, p.1-15, 2016.

NIE, S. et al. Above-ground biomass estimation using airborne discrete-return and full-waveform LiDAR data in a coniferous forest. Ecological Indicators, v.78, p.221-228, 2017.

NINGTHOUJAM, R.K. et al. Retrieval of forest biomass for tropical deciduous mixed forest using ALOS PALSAR mosaic imagery and field plot data. International Journal of Applied Earth Observation and Geoinformation, v.69, p.206-216, 2018.

PAGE, S.E. et al. Tropical peatland fires in Southeast Asia. In: Tropical fire ecology. Berlin: Springer Praxis Books, 2009.

PHUA, M.H. et al. Synergistic use of Landsat $8 \mathrm{OLI}$ image and airborne LiDAR data for above-ground biomass estimation in tropical lowland rainforests. Forest Ecology and Management, v.406, p.163-171, 2017.

R CORE TEAM. R: uma linguagem e ambiente para computação estatística. 2017.

SANTI, E. et al. The potential of multifrequency SAR images for estimating forest biomass in Mediterranean areas. Remote Sensing Environment, v.200, p.63-73, 2017.

SENTINEL-1 TEAM. Sentinel-1 user handbook. European Space Agency, 2013.

SENTINEL-2 TEAM. Sentinel-2 user handbook. European Space Agency, 2015.

SILVA, F.A.S.E.; AZEVEDO, C.A.V. The Assistat software version 7.7 and its use in the analysis of experimental data. African Journal of Agricultural Research, v.11, n.39, p.3733-3740, 2016.

URBAZAEV, M. et al. Estimation of forest aboveground biomass and uncertainties by integration of field measurements, airborne LiDAR, and SAR and optical satellite data in Mexico. Carbon Balance Management, v.13, n.1-20, 2018.

WILCOX, R. Robust regression. In: Introduction to robust estimation and hypothesis testing. 2017.

WORLD BANK GROUP. Forest area (\% of land area): Indonesia. 2018. Disponível em: https://data.worldbank.org/indicator/ AG.LND.FRST.ZS?end=2015\&locations=IDtart=2015\&type=sha ded\&view=map\&year $=2010$ 